\title{
COMPARISON OF ORGAN DOSES AND IMAGE QUALITY BETWEEN CT AND FLAT PANEL XPERCT SCANS IN WRIST AND INNER EAR EXAMINATIONS
}

\author{
J. Damet ${ }^{1, *}$, M. Sans-Merce ${ }^{1}$, F. Miéville ${ }^{1}$, M. Becker ${ }^{2}$, P. A. Poletti ${ }^{2}$, F. R. Verdun ${ }^{1}$ and S. Baechler ${ }^{1}$ \\ ${ }^{1}$ Institute of Radiation Physics, University Hospital Center and University of Lausanne, Grand Pré 1, 1007 \\ Lausanne, Switzerland \\ ${ }^{2}$ Department of Radiology, Radiodiagnostic Service, University Hospital of Geneva, Rue Gabrielle-Perret- \\ Gentil 4, 1211 Geneva 14, Switzerland \\ *Corresponding author: jerome.damet@chuv.ch
}

\begin{abstract}
The aim of this study was to evaluate and compare organ doses delivered to patients in wrist and petrous bone examinations using a multislice spiral computed tomography (CT) and a C-arm cone-beam CT equipped with a flat-panel detector (XperCT). For this purpose, doses to the target organ, i.e. wrist or petrous bone, together with those to the most radiosensitive nearby organs, i.e. thyroid and eye lens, were measured and compared. Furthermore, image quality was compared for both imaging systems and different acquisition modes using a Catphan phantom. Results show that both systems guarantee adequate accuracy for diagnostic purposes for wrist and petrous bone examinations. Compared with the CT scanner, the XperCT system slightly reduces the dose to target organs and shortens the overall duration of the wrist examination. In addition, using the XperCT enables a reduction of the dose to the eye lens during head scans (skull base and ear examinations).
\end{abstract}

\section{INTRODUCTION}

Diagnostic examinations for the identification of carpal ligamentous disruptions (e.g. rupture of the scapholunate ligament) are usually 2-fold and require the use of an X-ray installation and a computed tomography (CT) scan. Contrast enhancing agent is first injected in the wrist to assess the presence of dye leakage through the scapholunate joint with CT arthrography images ${ }^{(1)}$. The flat-panelbased XperCT Allura FD20 system ${ }^{(2)}$ (Philips Healthcare, Best, The Netherlands) enables performing the whole examination at once, thus reducing the overall duration of the examination and avoiding the need to move patients into another diagnostic room.

Diagnostic examinations for the identification of lesions or diseases in the petrous bone or for performing CT-guided interventional procedures of the skull base, such as fine needle aspiration biopsies, are also 2 -fold ${ }^{(3)}$. The first step is an X-ray radiography for screening purposes followed by a high-resolution multislice spiral CT examination. It is well known that head CT scans result in significant radiation exposure to the lens of the ey $\mathrm{e}^{(4)}$. Using the XperCT system may reduce the dose delivered to the eye lens.

The present study compares both techniques, for wrist and head scans, with regard to dose assessment and image quality. The aim was to evaluate and compare the quality of reconstructed images as well as doses delivered to patients using a Siemens Somaton Sensation 64 multislice spiral CT (Siemens
Healthcare, Erlangen, Germany) and a detector angiographic system, Allura FD 20(Philips Health Care, Best, The Netherlands). Measurements were performed under different XperCT operating modes. The dose to the target organ, i.e. the wrist or the petrous bone, together with those to most radiosensitive nearby organs, i.e. the thyroid and the eye lens, were measured and compared.

\section{METHODS}

\section{Dose measurements}

Measurements of radiation dose were performed on in-house-developed head and wrist phantoms using thermoluminescent detectors (TLDs) and a Radcal 3036 dosemeter with an external $11-\mathrm{cm}^{3}$ ion chamber. The hand phantom consisted of a human hand skeleton embedded in resin and a 4- $\mathrm{cm}$ thick Plexiglas $10 \times 10 \mathrm{~cm}^{2}$ plate. Two phantoms were used for the head: one with a skull embedded in transparent resin for the measurements of doses from scatter radiation to the thyroid and the eye lens and one made of an empty skull that can be opened to place detectors precisely on the petrous bone.

For the XperCT system, the X-ray tube and the flat-panel detector were mounted on a C-arm structure to provide radiographic projections over an angle of $240^{\circ}$.

Several operating modes were considered: 300 images per rotation, 600 images per rotation and 3D rotational angiography (3DRA) mode using 120 
images per rotation. For the multislice spiral CT, all measurements of radiation exposure on phantoms were performed using standard protocols for the wrist $(120 \mathrm{kV} / 250 \mathrm{mAs}, 0.18 \mathrm{~mm}$ reconstruction thickness on XperCT and $120 \mathrm{kV} / 195 \mathrm{mAs} /$ pitch of 0.9 , $0.4-0.2 \mathrm{~mm}$ reconstruction thickness on $\mathrm{CT}$ ) and petrous bone $(120 \mathrm{kV} / 250 \mathrm{mAs}, 0.18 \mathrm{~mm}$ reconstruction thickness on XperCT and $120 \mathrm{kV} / 430 \mathrm{mAs} / \mathrm{pitch}$ of $0.8,0.4-0.2 \mathrm{~mm}$ reconstruction thickness on $\mathrm{CT}$ ) examinations.

\section{Wrist examination}

Measurements were performed placing the Radcal ion chamber on the 4-cm thick Plexiglas plate. Further measurements were done using TLD bands all over the human hand phantom.

\section{Petrous bone examination}

For the XperCT system, measurements were performed with both head phantoms by positioning the Radcal ion chamber on the ear and inside the skull as close as possible to the petrous bone (Figure 1). For the CT scanner, measurements were done using the resin head phantom. As for the case of the wrist examination, dose measurements to nearby radiosensitive organs, i.e. lens and thyroid, were performed.

\section{Image quality measurements}

The comparison of the image quality of both systems was based on measurements with the Catphan 600 phantom $^{(5)}$ (The Phantom Laboratory, Salem, NY, USA). Images of the $0.28-\mathrm{mm}$ tungsten carbide bead of the CTP528 high-resolution module were used to evaluate the spatial resolution and calculate the modulation transfer function (MTF) using an inhouse-developed Matlab77 (The MathWorks, Natick, MA, USA) program. In addition, two attending radiologists subjectively evaluated the image quality of patients examined with both systems. Parameters are detailed in Figures 4 and 5.

\section{RESULTS}

\section{Dose measurements}

Doses delivered to target and nearby organs are given in Table 1 for wrist $\mathrm{CT}$ and XperCT scans and in Table 2 for head CT and XperCT scans.

\section{Image quality measurements}

Reconstructed images of the Catphan point source are shown in Figure 2. The images from both systems are reconstructed in the axial, coronal and sagittal planes to obtain a 3D visualisation of

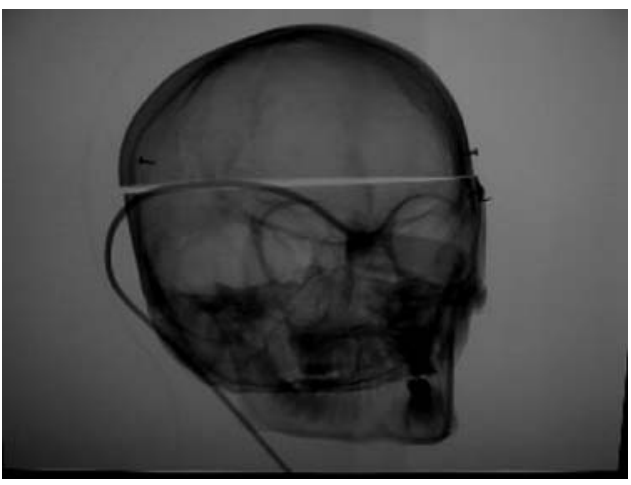

Figure 1. Reconstructed 3D image of the empty skull phantom with the XperCT system showing the position of the Radcal ion chamber close to the petrous bone.

Table 1. Dose measurements (in mGy) for wrist examinations.

\begin{tabular}{lcccc}
\hline $\begin{array}{l}\text { Operational } \\
\text { modes }\end{array}$ & \multicolumn{3}{c}{ XperCT } & Multislice \\
\cline { 2 - 4 } & $\begin{array}{c}300 \\
\text { images } \\
\text { per rot }\end{array}$ & $\begin{array}{c}600 \\
\text { images } \\
\text { per rot }\end{array}$ & 3DRA & \\
\hline Wrist (4 cm plate) & 23.9 & 47.8 & 0.5 & 62.3 \\
Lens & 0.24 & 0.47 & - & 0.14 \\
Thyroid & 0.04 & 0.08 & - & 0.006 \\
Wrist (phantom) & 22.1 & 44.1 & 0.6 & 62.3 \\
\hline
\end{tabular}

Table 2. Dose measurements (in mGy) for petrous bone examinations.

\begin{tabular}{lcccc}
\hline $\begin{array}{l}\text { Operational } \\
\text { modes }\end{array}$ & \multicolumn{3}{c}{ XperCT } & $\begin{array}{c}\text { Multislice } \\
\text { CT }\end{array}$ \\
\cline { 2 - 3 } & $\begin{array}{c}\text { 300 images } \\
\text { per rot }\end{array}$ & $\begin{array}{c}\text { 600 images } \\
\text { per rot }\end{array}$ & 3DRA & \\
\hline Petrous bone & 17.6 & 35.2 & 3.4 & 95.6 \\
Lens & 9.3 & 18.4 & 1.5 & 39.5 \\
Thyroid & 1.0 & 1.9 & 0.2 & 2.0 \\
\hline
\end{tabular}
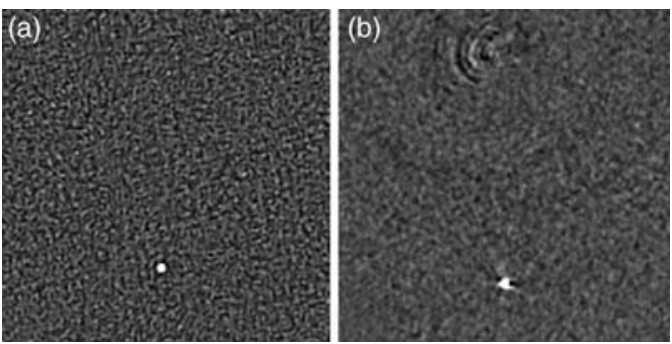

Figure 2. Reconstructed images from data acquired with the Siemens multislice CT (a) and the Philips XperCT (b) using standard protocols for wrist examinations. 


\section{J. DAMET ET AL.}

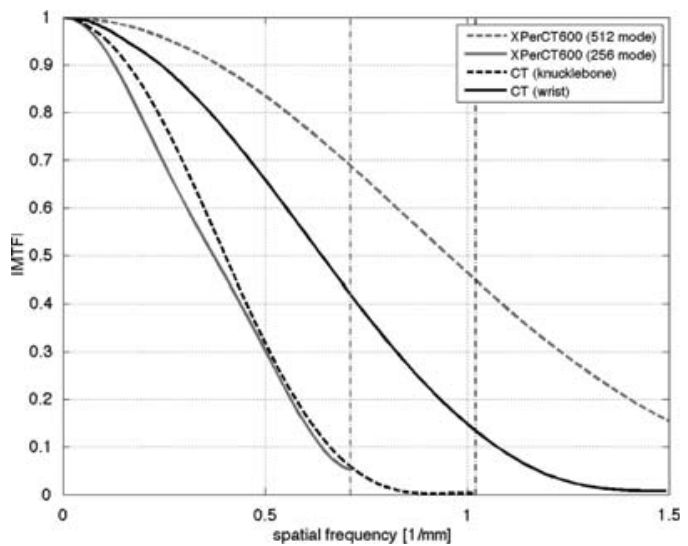

Figure 3. MTF of both systems for wrist and petrous bone examination protocols with different acquisition modes. organs. The axial projection was used to calculate the in-plane MTF (Figure 3).

Images of a patient who underwent an arthrography of a post-traumatic wrist with the XperCT system, and shortly after, with the CT scan, were evaluated (Figure 4). Axial CT and XperCT reformation images of a patient admitted after a facial trauma (Lefort III fracture) were obtained from the initial full head acquisition to rule out a committed fracture of the petrous bone (Figure 5). In both cases, the Ultra High Resolution Mode for bone was used. Complete diagnostic could be performed using either system.

\section{DISCUSSION}

\section{Dose comparison}

For both types of examination, the measured dose delivered to all organs using the XperCT system is
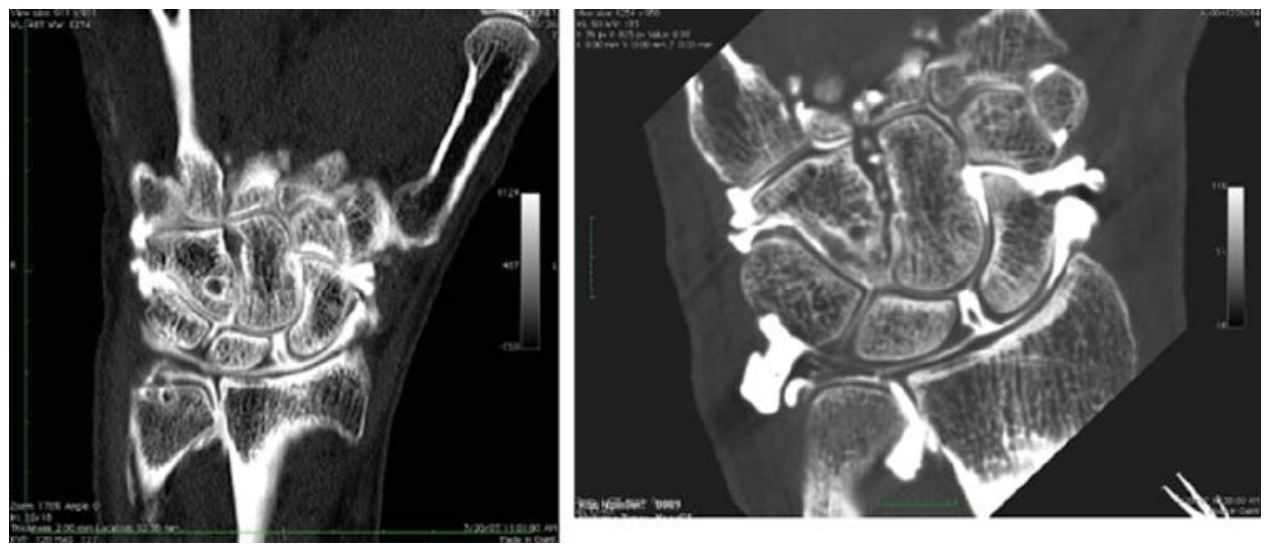

Figure 4. Images of a patient admitted after a wrist trauma. A rupture of the scapholunate ligament can be identified on CT (120kV/127mAs/MPR reconstruction) (left) and XperCT (120kV/250mAs) (right) images.
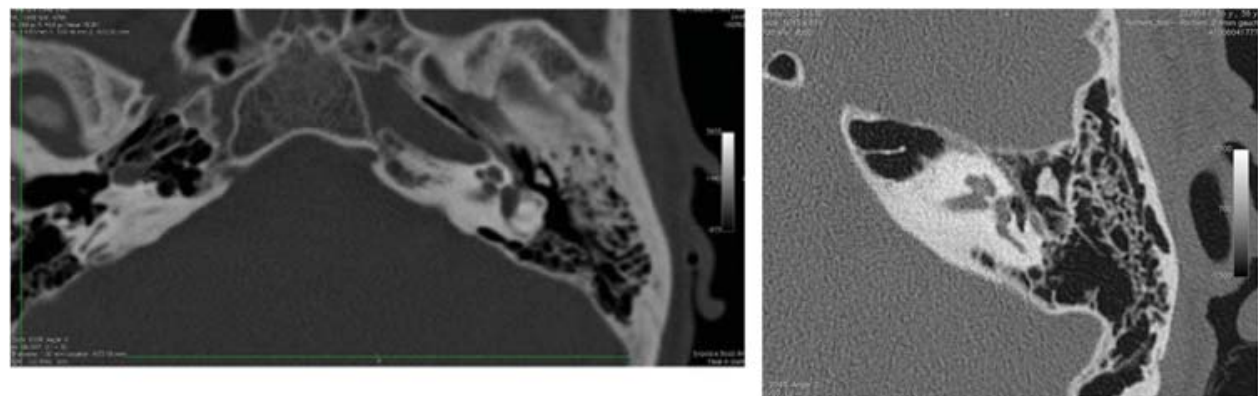

Figure 5. CT (120kV/300mAs) (left) and XperCT $(120 \mathrm{kV} / 250 \mathrm{mAs})$ (right) reconstructed images obtained from the raw data of the initial full head scan. 


\section{ORGAN DOSES AND IMAGE QUALITY}

multiplied by two when doubling the number of images per rotation (from 300 to 600 images per rotation). Using the 3DRA fluoroscopy mode of the XperCT (120 images per rotation, $63 \mathrm{kV}$ and 59 $\mathrm{mAs}$ ) drastically reduces the doses, up to a factor of 100 for the wrist when comparing with the 600 images per rotation mode (Table 1).

Using the human hand phantom shows a slightly lower dose to the wrist compared with the 4-cm thick Plexiglas plate. The dose to the wrist based on the CT scan is higher by a factor of 1.3 and 2.6 compared with the XperCT scan based on the 600 and 300 images per rotation mode, respectively. Concerning nearby radiosensitive organs in case of wrist examinations, the dose to the eye lens and the thyroid is lower when using the CT scanner. Considering the 300 images per rotation mode, a reduction factor of 1.7 and 6.7 is observed for the eye lens and the thyroid, respectively. Dose differences to nearby organs are explained by the distinct beam collimation of the CT scanner (fan beam) and the XperCT system (cone beam). This effect is even more pronounced for the thyroid, which is located at a larger distance from the region under examination.

The measured dose to the petrous bones using the resin embedded skull phantom is higher than doses measured with the empty skull phantom. This is due to the back scattering produced by the resin and the fact that the detector is placed on the surface of the skull. The dose delivered to the petrous bone is 2.7 and 5.4 times higher for the CT examination compared with the XperCT examination realised with the 600 and 300 images per rotation mode, respectively. Doses to the eye lens and the thyroid, which were obtained with the resin embedded skull phantom, are higher for the CT scan. Indeed, those organs are in the direct vicinity of the beam during the petrous bone examination. The dose delivered with the CT scan examination is increased by a factor of 4.3 for the eye lens and 2.0 for the thyroid in comparison with the examination done with the XperCT system using the 300 images per rotation mode.

The results show that for both examinations the dose to the target organ is lower when using the XperCT system compared with the CT scan. The dose is at least reduced by a factor of 1.3 for the wrist and 2.6 for the petrous bone.

\section{Image quality comparison}

The reconstructed images of the Catphan 600 phantom shown in Figure 2 present more artefacts with the XperCT system than with the CT scanner. This can be explained by the incomplete rotation of the $\mathrm{C}$-arm structure $\left(240^{\circ}\right.$ vs. $360^{\circ}$ for CT scan) and by the fact that it has significantly more beam hardening due to specific filters designed to compensate for absorption differences in objects and enhance the image quality for cerebral and lower peripheral angiography. The energy shift towards the highest values of the spectrum induces a diminution of the attenuation for high-density objects like bones. The spatial resolution depends on the resolution matrix and the field of view. Two matrices are used with the XperCT system. The 256 reconstruction mode - default mode-(XperCT600-256 curve in Figure 3) is appropriate for visualising soft tissue structures and low-contrast regions. A higher spatial resolution is available using the 512 reconstruction mode (XperCT600-512 curve on Figure 3) of the XperCT system that would be more suitable for objects with high contrast since the noise level is higher. The spatial resolution of the CT scanner is limited by the matrix size, which is fixed at $512 \times$ 512. The size of the pixels is therefore larger for a small region of interest. The MTF of the images of the petrous bones (Figure 3) is thus below the MTF of the images of the wrist (Figure 3). The reconstructed images for examinations done with the CT scanner have a lower spatial resolution in the axial axis but a higher contrast resolution than images acquired with the XperCT system.

According to the subjective evaluation of attending radiologists, both systems provide equivalent diagnostic image quality and information for wrist examinations. Concerning petrous bone examinations, although the ossicular chain and the inner ear were depicted more clearly on the CT scanner, the XperCT system depicted all essential details related to an examination of the petrous bone, such as incudo-stapedial joint, round and oval windows, cochlear turns, canal of the intermediate nerve and the tegmen tympani.

\section{CONCLUSION}

The measured dose to the target organs, i.e. the wrist and the petrous bones, is higher when the examination is performed with the CT scanner compared with the dose for the same examination performed with the XperCT system. For wrist examinations, the dose to the nearby critical radiosensitive organs is higher with the XperCT system. On the contrary, for petrous bone examinations, the dose to nearby organs, mainly the eye lens, is lower with the XperCT system. For wrist examinations, using the XperCT scan shortens the time between the arthrography and the XperCT scan, and thus reduces diffusion of the contrast agent in the soft tissues.

The high-spatial resolution of the XperCT system enables high quality imaging of small structures, but the reconstructed images include artefacts and a high level of noise compared with the images 


\section{J. DAMET ET AL.}

obtained with the multislice CT scanner. The quality of reconstructed images from both systems guarantees adequate accuracy for diagnostic purposes for wrist and petrous bone examinations.

\section{ACKNOWLEDGMENTS}

The authors are grateful to Christel Elandoy, Olivier Brina and Patrice Bregis for their contribution to measurements and fruitful discussions.

\section{REFERENCES}

1. Lanz, U. and Schmitt, R. Diagnostic Imaging of the Hand, p.25, 2008 Thieme Publishing Group, Stuttgart,
Germany and Thieme New York, NY, USA, ISBN 9783-13-140581-4.

2. Söderman, M., Babic, D., Holmin, S. and Andersson, T. Brain imaging with a flat detector C-arm technique and clinical interest of XperCT. Neuroradiology 50, 863-868 (2008).

3. Greess, H., Baum, U., Römer, W., Tomandl, B. and Bautz, W. CT und MRT des Felsenbeins. HNO 50, 906-919 (2002).

4. Moström, U., Ytterbergh, C. and Bergström, K. Eye lens dose in cranial computed tomography with reference to the technical development of CT scanners. Acta. Radiol. Diagn. 27, 599-606 (1986).

5. Catphan® 500 and 600 Manual. (2009), The Phantom Laboratory Incorporated, Salem, NY 12865-0511, USA. 